\title{
PATTERN AND SPECIFICITY OF AXONAL OUTGROWTH FOLLOWING VARYING DEGREES OF CHICK LIMB BUD ABLATION ${ }^{1}$
}

\author{
KATHRYN W. TOSNEY ${ }^{2}$ AND LYNN T. LANDMESSER ${ }^{3}$ \\ Physiology Section, The Biological Sciences Group, The University of Connecticut, Storrs, Connecticut 06268
}

Received January 9, 1984; Revised May 14, 1984; Accepted May 16, 1984

\begin{abstract}
Motoneurons grow into the chick hindlimb via consistent pathways, within which they make specific decisions leading to their correct targets. To determine which axonal guidance features dictate the position of the pathways and to examine the distribution of specific cues, we totally or partially ablated the early hindlimb bud and determined how the subsequent pattern of nerve outgrowth related to the distribution of tissue remnants.

Our results suggest that local elements determine the gross anatomical pattern of outgrowth. First, determinants of individual pathways could be selectively removed without altering the pattern in other regions. Second, neurites were restricted to the plexus region at the base of the leg (within which, for unknown reasons, they proceeded posteriorly) unless distal permissive pathways or nearby target remnants were present. Finally, we found that the central region of the pelvic girdle, adjacent to the plexus region, determines the position where the major nerve trunks enter the leg. When gaps were introduced in this region of the girdle, nerves traversed the gaps and directly innervated adjacent muscle. The developing girdle is probably a nonpermissive environment for axon elongation, and axons enter the leg only where it is locally absent.

Our results also support the concept that the specific cues that neurites use to reach their appropriate muscles are local. We find that neurites could make correct and specific decisions in the plexus region in the absence of all tissues distal to the pelvic girdle. This shows that the cues for these decisions are independent of the target and must reside in the local mesenchyme. In addition, when muscle remnants were present they were correctly innervated. This suggests that the target or mesenchyme associated with the target exerts a specific, short-range attraction.
\end{abstract}

During development, motoneurons generate a pattern of innervation in the avian hindlimb that is complex, consistent, and precise. The motoneurons are specified for particular targets before nerve outgrowth (Lance-Jones and Landmesser, 1980b; Landmesser, 1980). During outgrowth, neurites from different motoneuron pools intermix proximally and then segregate to take up characteristic positions in the plexi and nerve trunks (Lance-Jones and Landmesser, 1981a). The pattern of neurite outgrowth is similar in form to the adult pattern and is specific and precise (Landmesser and Morris, 1975; Landmesser, 1978a, b) before the major period of naturally occurring motoneuron cell death (Hamburger, 1975; Chu-Wang and Op-

\footnotetext{
${ }^{1}$ We thank V. Argiro, L. Dahm, G. Pilar, and J. Tuttle for critical evaluation of the manuscript and B. Wolmer for secretarial aid. This work was supported by National Institutes of Health Grant NS 19640 and by Muscular Dystrophy Association and National Institutes of Health postdoctoral fellowships.

${ }^{2}$ Present address: Division of Biological Sciences, The University of Michigan, Ann Arbor, MI 48109.

${ }^{3}$ To whom correspondence should be addressed, at The Biological Sciences Group, U-42, The University of Connecticut, Storrs, CT 06268 .
}

penheim, 1978) and prior to muscle individualization (Romer, 1927).

The gross morphology of the innervation pattern is, to some degree, separable from the specificity of innervation. For instance, when the spatial relationships between the cord and leg are altered early in development, the subsequent pattern of innervation can be grossly normal morphologically, although muscles are often innervated by an incorrect complement of motoneurons (Morris, 1978; Hollyday, 1981; Lance-Jones and Landmesser, 1981b). This suggests that neurites are constrained to take certain pathways in the limb regardless of their identity.

Lewis et al. (1981) have shown that the major nerve trunks form in the absence of muscle, but it is unknown which other elements in the limb contribute to the establishment of the gross anatomical nerve pattern. Therefore, we totally or partially removed the hindlimb bud before nerve outgrowth, reconstructed the subsequent nerve pattern, and determined how outgrowth related to the pattern of tissue remnants.

We were also interested in the specific cues that motoneurons use to reach the appropriate muscles within the constraints imposed by the gross nerve pattern. A number of previous studies have suggested that such cues are relatively local. Thus, 
when motoneurons are displaced moderate distances early in development, their growth cones are able to alter their trajectories within the plexus region and reach their appropriate muscle (Lance-Jones and Landmesser, 1980b; Ferguson, 1983). Following more extreme displacements, however, motoneurons usually innervate inappropriate muscles, unless the foreign nerve trunks in which they are traveling happen to come sufficiently close to their original target region (Lance-Jones and Landmesser, 1981b; Summerbell and Stirling 1981; LanceJones, 1982; C. Lance-Jones, personal communication.

However, the important cues may not be produced locally as neurites could respond to local portions of a gradient that is produced by a distant source-for instance, the target itself. To determine whether specific pathway choice is dominated by distant or local tissues, we examined the specificity and direction of travel of neurites in embryos in which some or all of the normal targets and peripheral pathways had been removed by early hindlimb bud ablations.

\section{Materials and Methods}

Embryonic surgery. White Leghorn eggs of 2 to 4 days incubation were candled and $1 \mathrm{~cm}^{2}$ of shell was removed from.above the embryo with a dental drill. The vitelline and amniotic membranes over the posterior part of the embryo were torn open and the area was stained with a drop of $0.5 \%$ sterile neutral red in saline solution. The right hindlimb bud of stages (Sts) 16 to 26 (Hamburger and Hamilton, 1951) embryos was removed with sharpened tungsten needles. The proximodistal level and angle of the incision were varied to leave a range of proximal thigh tissues in different embryos. The eggs were sealed with molten paraffin and a flamed coverslip and were incubated until the desired stages.

Eighty-one of the embryos (53\%) survived the operation. Of these, the leg was removed from 38 embryos during the period of motoneuron birth (Sts 16 to 18), from 37 embryos during the period of initial neurite outgrowth (Sts 19 to 22 ), and from 5 embryos after the neurites had entered the limb (Sts 23 to 26). There were no substantial differences in the resulting nerve patterns that correlated with the stage at which the operation took place. Nineteen embryos were killed for examination during early innervation, before motoneurons have migrated to their final position within the cord (Sts 23 to 27), and 62 embryos were killed at Sts 28 to 32 , before completion of the normal period of motoneuron cell death (Hamburger, 1975). Horseradish peroxidase (HRP) was injected into 75 of the embryos; an additional 6 embryos were fixed without dissection to determine whether the nerves invaded tissues that we normally removed during our HRP injections. They did not. The nerve patterns were reconstructed from camera lucida drawings (as in Lance-Jones and Landmesser, 1980a) or determined by close examination of sectioned embryos.

Injection with HRP. Operated embryos were decapitated, eviscerated, and placed in an oxygenated bath of Tyrode's solution at room temperature $\left(20\right.$ to $\left.22^{\circ} \mathrm{C}\right)$. A ventral laminectomy was performed, and the overall pattern of nerve projection was determined by examination. A solution of $10 \%(\mathrm{w} / \mathrm{v}) \mathrm{HRP}$ in Tyrode's was pressure injected into a single segment of the cord or into a small nerve branch in the periphery in both experimental and control sides. HRP injected in this matter intensely stains the neurites and somata of a proportion of the neurons present at the injection site. Diffuse (nongranular) HRP reaction product fills the entire extent of the cell, similar to intracellular IIRP injections. This allows both the trajectory and the identity of the neurites to be determined. Since only damaged axons or cell bodies take up the stain in this manner (Lance-Jones and Landmesser, 1981a), even if HRP spreads from the injection site, it does not label cells in this manner. Thus, very confined injections can be made, and if motoneurons in an adjacent segment are inadvertently labeled, this is obvious from the position of the labeled somata in the cord.

Following injection and incubation for 4 to $5 \mathrm{hr}$ at $3 \mathrm{l}$ to $34^{\circ} \mathrm{C}$ to allow HRP transport, embryos were fixed, washed thoroughly in Tris buffer, processed with diaminobenzidine, paraffin embedded, and sectioned as described by Lance-Jones and Landmesser (1980a). In embryos older than St 27 , the positions of labeled cell somata in the cord were reconstructed as in Landmesser $(1978 \mathrm{a}, \mathrm{b})$.

Identification of tissue remnants in leg-ablated embryos. The tissue type was identified by histological staining characteristics. In material processed for HRP visualization and counterstained with cresyl violet, condensed chondrocytes stained dark blue to purple, while muscle remnants were lighter blue. Twenty-four operated embryos were stained with Alcian blue following the protocols of Derby and Pintar (1978) to enhance visualization of skeletal and muscle remnants. The Alcian blue method we used stains areas rich in glycosaminoglycans; this allowed us to distinguish regions of precartilage, even before overt chondrogenesis. Eighteen of these were also processed for HRP visualization. Counterstaining with eosin stained muscle remnants bright pink; dilute hematoxylin counterstain allowed us to identify myotubes as cells with multiple nuclei. Dehydrated, cleared slides were mounted in Permount.

The spatial relationships among tissue remnants were determined with greatest facility when the embryos were sectioned perpendicular to the long axis of the spinal cord. The relative positions of cartilage and muscle remnants and nerves were reconstructed in 27 embryos sectioned in this plane, and the identity of muscle remnants was determined by their position.

\section{Results}

The normal gross anatomical nerve pattern. The major features of a normal nerve pattern in the chick hindlimb are shown in Figure 1 for comparison with the results reported below. Along the anterior-posterior (A-P) axis, the spinal nerves meet in the plexus region at the base of the limb and enter the leg as two major nerve trunks, the anterior crural and the posterior sciatic. The crural plexus supplies primarily the preaxial thigh, while the sciatic trunk supplies postaxial thigh and all of the shank and foot. Along the dorsal-ventral (D-V) axis, each of these major nerve trunks diverges into dorsal and ventral branches. The crural trunk branches at the level of the plexus to form dorsal crural and ventral obturator nerve trunks, and the sciatic branches at the level of the proximal thigh to form dorsal peroneal and ventral fibular trunks. Within the leg proper, muscle and cutaneous nerves leave the nerve trunks at characteristic positions.

The gross anatomical pattern following hindlimb bud ablation. In these experiments limbs were ablated either totally or partially, providing a series of embryos with a range of distal structures. With the most complete ablations the leg was dramatically reduced; muscles and bones were absent, the pelvic girdle was incomplete or absent, and loosely packed mesen-

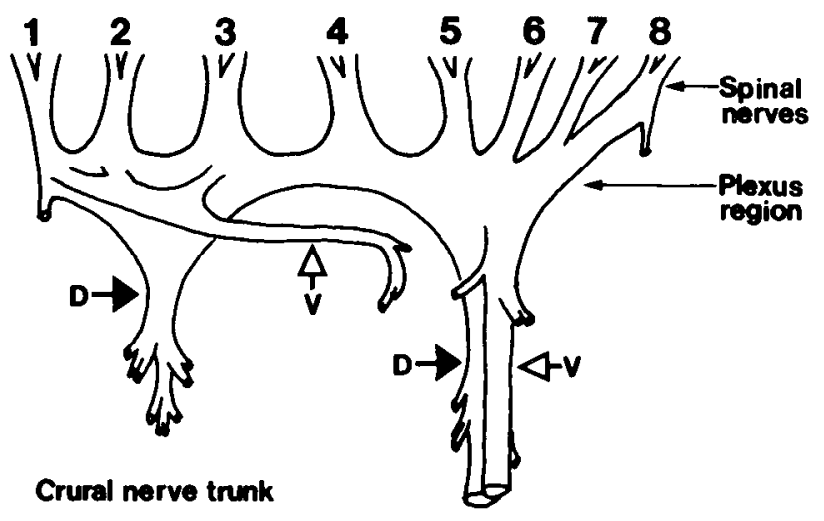

\section{Sciatic nerve trunk}

Figure 1. Major features of the normal nerve pattern. A schematic reconstruction of a normal nerve pattern from the ventral side is shown. The spinal nerves from LS 1 to 8 converge to meet in the plexus region and enter the leg as two major nerve trunks, the crural in the anterior and the sciatic in the posterior. The anterior trunk branches at the level of the plexus to form a dorsal crural trunk ( $D$; solid arrow), which enters the leg directly, and a ventral obturator trunk ( $V$; open arrow), which courses ventrally and posteriorly before entering the leg posterior to the femur. The sciatic trunk has similar dorsal $(D)$ and ventral $(V)$ branches. Muscle nerves leave the nerve trunks at characteristic positions in the leg proper. 


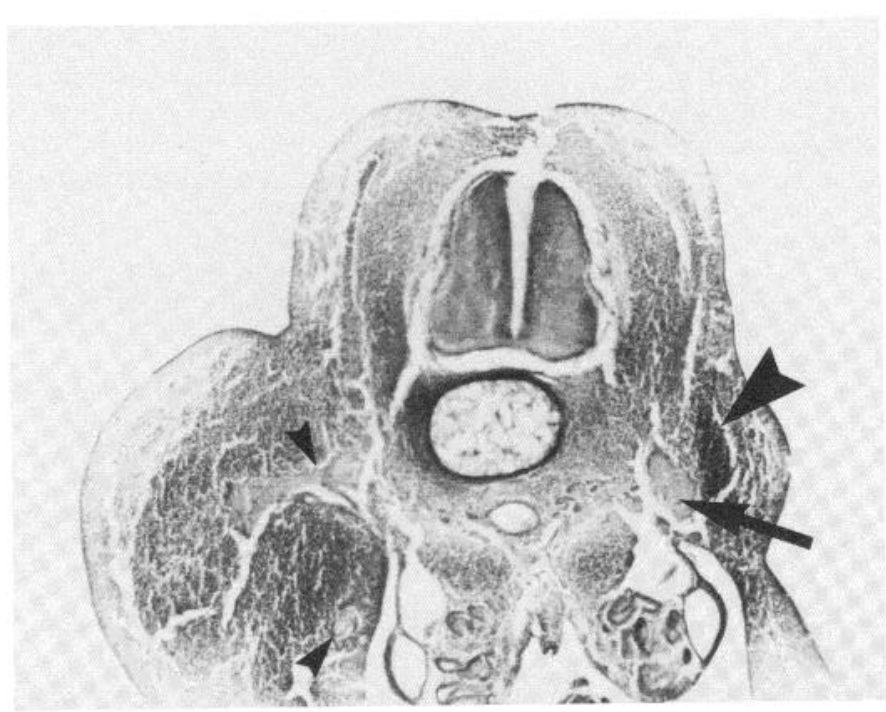

Figure 2. Radical leg ablation. A cross-section at level LS2 through a St 29 embryo in which the right hindlimb bud was removed at St 18 is shown. On the normal side (at left), the dorsal crural and ventral obturator nerve trunks enter the leg (small arrowheads). On the right side, the ventral root ends in an enlarged "neuroma" within the plexus area (arrow). Nerves have not penetrated the available mesenchyme that surrounds the plexus region. Leg tissue is restricted to a portion of the dorsal girdle (large arrowhead) and covering ectoderm. Loosely packed mesenchyme fills the area between the girdle remnant and the ectoderm. A portion of the body wall can be seen ventral to the leg remnant. Alcian blue and eosin stain.

chyme filled the area between the girdle remnant and the limb epithelium (Fig. 2). The nerve pattern in these embryos was characteristically simple; the ventral roots joined in their normal position, ventral-lateral to the dermatome, and formed a single, elongated plexus (Fig. $3 A ; N=18$ ). As noted by Hamburger (1958), this plexus forms in exactly the same position as would a normal plexus, and with the most radical ablations, few if any axons appear able to leave this plexus region and invade adjacent mesenchyme (see also Oppenheim et al., 1978). Although this structure has been called a neuroma in previous studies, we prefer the term plexus since we will show that neurites do not end blindly in this region. Axial nerves occasionally extended in a quasisegmental manner from the plexus into thoracic and body wall muscles.

When remnants of leg muscles and associated tissue remained in the embryo, nerve trunks extended into the leg from the plexus area. The nerve trunks, with exceptions to be discussed below, were in their normal positions and formed independently of one another. When only anterior muscle remnants remained, a crural nerve trunk formed (Fig. $3 B ; N=8$ ); when only posterior muscles remained, a sciatic nerve trunk formed (Fig. $3 C ; N=2$ ); and when even small muscle remnants were present in both compartments, both nerve trunks were represented (Fig. $3 D ; N=34$ ). When the sciatic nerve trunk was present, it ended at the site of limb truncation. The dorsal and ventral branches of the crural trunk also formed independently, in some cases only the obturator or dorsal crural trunk being present (Table I; see also Figs. $3 B$, 8, and 9). Evidently, the elements that determine the gross anatomical pattern are local, and one determinant can be removed without greatly altering the nerve pattern at other positions.

The normal pattern of anterior and posterior nerve trunks was clearly altered in one subset of leg-ablated embryos. In these, peripheral nerves left the plexus centrally, between the normal nerve trunks (Fig. $3 E$, arrow, and Fig. $4 ; N=19$ ). We completely reconstructed the nerve and tissue patterns in 27 operated embryos sectioned perpendicular to the spinal cord to
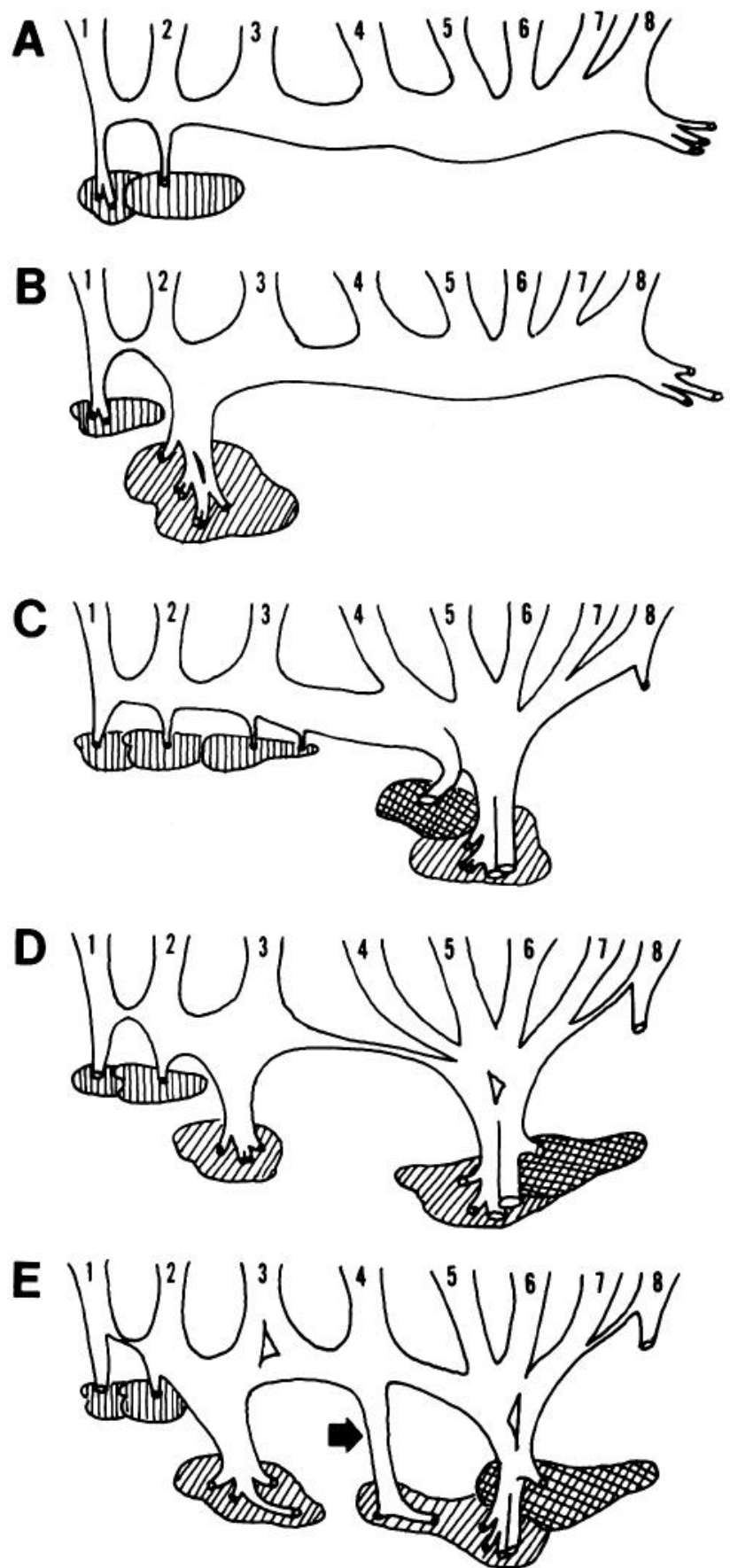

Figure 3. The relationship between nerve outgrowth patterns and muscle remnants. $A$, Leg muscle remnants are absent in this radically ablated leg. The ventral roots meet in the plexus area to form an enlargement that has been termed a neuroma (see also Fig. 6). In the anterior, axial nerves leave the plexus area to branch within body wall muscles. $B$, Muscle remnants are present only in the dorsal anterior compartment of this partially ablated leg. A dorsal crural nerve trunk has formed; all other neurites remain within the plexus area. $C$, When we ablated all anterior muscles, leaving only posterior muscle remnants, a representative of the posterior, sciatic nerve trunk formed, but a crural nerve trunk was absent. Small axial nerves to the body wall muscles also formed as in more radical ablations $(A)$. $D$, When even small muscle remnants were left in both anterior and posterior limb compartments, representatives of both anterior and posterior nerve trunks formed. $E$, In some cases novel nerves formed centrally (arrow), between the normal entry point of the anterior and posterior nerve trunks. In several cases, the distribution of muscle remnants was indistinguishable from that seen in embryos with more normally positioned nerve trunks (compare to $D$ ). Diagonal lines, dorsal muscle; hatched lines, ventral muscle; vertical lines, axial muscle. 
TABLE I

Independent formation of dorsal and ventral nerve trunks The obturator (OBT) and crural (CRU) nerves were identified by position in 27 completely reconstructed embryos. In 10 cases, one nerve formed in the absence of the other. Whenever the dorsal crural nerve trunk formed, muscle was present in the dorsal-anterior compartment of the leg. The presence (+) or absence $(-)$ of each nerve is indicated.

\begin{tabular}{ccc}
\hline No. of Cases & OBT & CRU \\
\hline 5 & - & - \\
8 & - & + \\
2 & + & - \\
12 & + & + \\
\hline
\end{tabular}

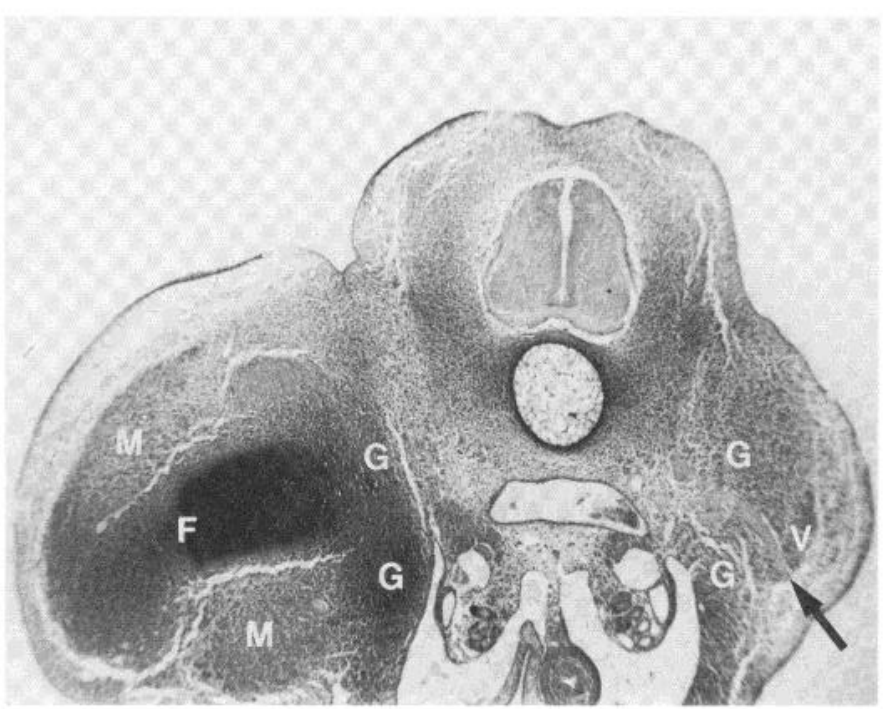

Figure 4. Novel central nerves. A cross-section of a St 29 embryo in which the right leg bud has been partially ablated is shown. In the normal left leg at this level, dorsal and ventral muscles $(M)$ are separated by the femur $(F)$ which articulates with the more medial pelvic girdle $(G)$. Note that at this level the pelvic girdle extends across the full dorsal-ventral base of the leg. On the experimental side, only a small ventral muscle rudiment is present $(V)$. The femur is absent, and the pelvic girdle is incomplete. A small nerve (arrow) leaves the plexus area and directly innervates the muscle remnant through a small gap in the central pelvic girdle.

determine whether the absence of a particular structure correlated with the formation of these central branches. Novel central nerves were present in 12 of these embryos.

While peripheral muscle remained in all embryos with novel central nerves, the identity of the muscle remnants (Fig. $3 E$ ) differed from specimen to specimen. This suggests that particular muscle groups were not important in the establishment of the novel pathways. In contrast to the suggestion of Bennett et al. (1980), the central nerves do not appear to be formed by a nonspecific chemotactic response to a predominance of central muscle tissue.

We were also able to rule out a significant role for the femur and the dorsal and ventral girdle elements in preserving the normal nerve pattern. The femur was absent in 5 embryos, and in 4 of these, normal anterior and posterior nerve trunks formed. In addition, 3 of the 11 embryos that retained remnants of a femur had novel central nerves.

However, the precursor of the girdle appeared to play an important role in restricting nerves and thereby contributing to normal plexus and nerve trunk formation. Although the girdle has not undergone chondrogenesis at the time of plexus and nerve trunk formation, the rough extent of its precursor can be visualized by Alcian blue staining, since it is rich in glycosaminoglycans. We found that neither the dorsal part of this precursor (equivalent to most of the ilium) nor the ventral part (equivalent to the pubis and ischium) were important. In 12 embryos with novel central nerves, 8 had intact dorsal girdle elements and 4 had intact ventral elements.

In contrast, the central girdle, corresponding to the ventral part of the ilium between the pubis and the ischium, was deficient in all 12 cases where central nerves were found (cf. Fig. 5, $A$ and $B$ ). This region of the girdle precursor lies immediately distal to the plexus, between the areas where sciatic and crural nerve trunks form. In its absence, nerves left the plexus and directly innervated adjacent muscle; when it was present, nerves traveled posteriorly to their normal entry point into the limb and curved back to innervate muscle that lay opposite the central girdle fragment. This normal but roundabout innervation pattern was seen even when dorsal or ventral portions of the girdle precursor were absent. For example, the nerves in these cases did not take the shortest route to the muscle but followed the central girdle fragment toward the posterior (Fig. $5 \mathrm{C}$ ). These results suggest that the central
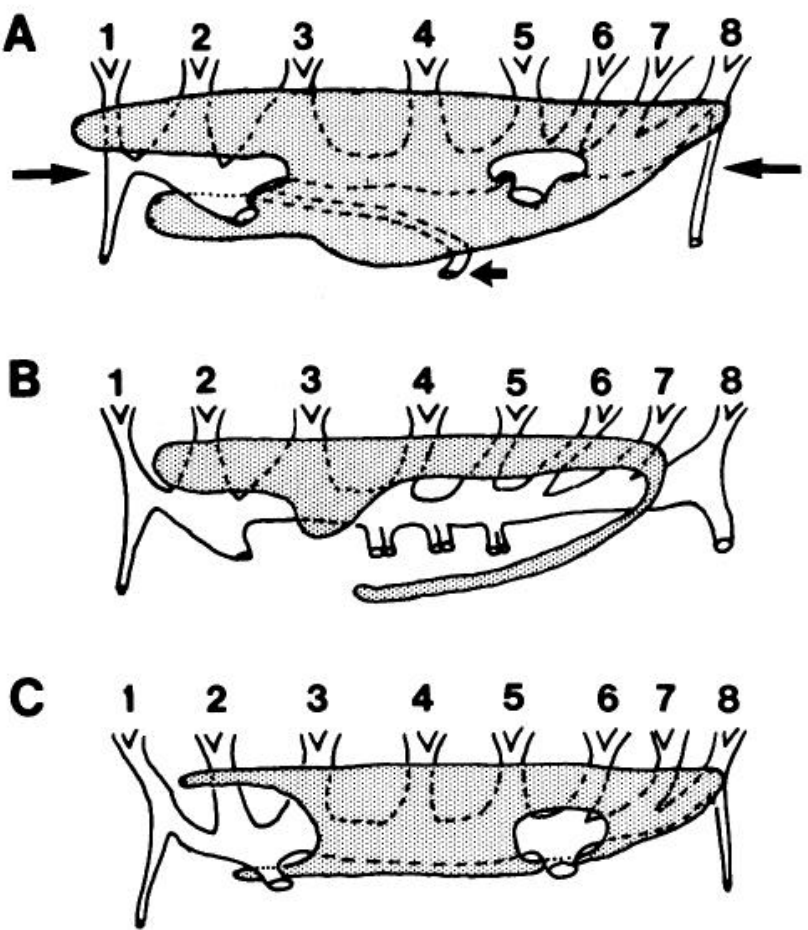

Figure 5. Relationship between the nerve pattern and the pelvic girdle precursor. $A$, Schematic reconstruction of the normal relation between the pelvic girdle precursor (stippled area) and the nerve pattern. The view of the girdle is from the limb side. The anterior and posterior nerve trunks enter the limb through hiatuses in the central region of the girdle. The normal plexus region lies close to the central axis of the girdle, on its medial side, in the plane indicated by the long arrows. The lumbosacral levels are indicated by numbers above the girdle. The obturator nerve (short arrow) courses posteriorly along the ventral portion of the girdle before entering the limb. $B$, When there is an experimentally produced hiatus in the central girdle precursor, nerves leave the plexus and directly innervate adjacent muscle. In the embryo illustrated, stippling indicates the girdle remnant. A single posterior nerve trunk in not formed; instead, three nerve trunks leave the plexus region and directly innervate the adjacent muscle. In the anterior compartment, a dorsal crural trunk is present, but the obturator nerve is absent, as it generally is when the ventral pelvic girdle is disrupted. $C$, When the central girdle precursor is complete, novel nerves are not formed, even when the dorsal or ventral girdle elements are depleted. In the reconstruction shown, the ventral girdle presursor is greatly depleted. Even so, nerves enter the limb via the normal anterior and posterior nerve trunks. They do not travel ventral to the girdle, which would provide the shortest pathway to the muscles left in the limb. The obturator nerve is also absent. 
girdle region normally prevents nerves from entering the leg centrally and serves to restrict the neurites to the plexus region, where they extend toward the posterior and enter the sciatic nerve trunk through a natural gap in the central girdle.

We have as yet been unable to correlate the formation of the main dorsal and ventral sciatic nerve trunks with similar obvious topographical features. However, we did notice that the obturator nerve failed to form whenever a ventral portion of the girdle precursor, corresponding to the ventral ilium and the ischium, was missing (see, for example, Fig. $5, B$ and $C$ ). It is probable that this portion of the girdle or the associated mesenchyme guides the obturator neurites toward their normal entry point in the limb and is therefore essential for the formation of this major nerve trunk. Of greater interest is the observation that, when the ventral girdle was present, the obturator often formed in the absence of its target muscles (11 cases). This shows that the target is not required for major nerve trunk formation, confirming the results of Lewis et al. (1981) on muscleless wings.

Specificity of growth and axonal sorting in leg-ablated embryos. Normally, the axons from different motoneuron pools are extensively intermingled in the proximal spinal nerves, but by the time they leave the plexus they have sorted out to occupy characteristic positions based on their identity (Lance-Jones and Landmesser, 1981a). In the absence of the limb, the neurites appear to end blindly in the plexus region in a neuroma (Hamburger, 1958; Oppenheim et al., 1978). By injecting small subsets of neurons with HRP, we were surprised to detect an unsuspected orderliness in the outgrowth pattern. All of the neurites in the plexus were found to travel toward the posterior (Fig. 6). This bias for posterior growth is not an aspect of development in unperturbed limbs: some neurites from all lumbosacral cord segments normally project anteriorly in the plexus, crossing over other axons destined for more posterior structures.

The bias for growth toward the posterior in embryos without leg muscle remnants was evident shortly after neurites had entered the plexus region and continued until St 32 , the oldest stage examined (Fig. 6). Neurites entered the plexus by St 23, as in normal development. By St 25, when the major nerve branches in the limb would normally be forming, neurites had traveled up to $200 \mu \mathrm{m}$ in a caudal direction in the plexus region. By St 27, the neurites had traveled $400 \mu \mathrm{m}$ toward the posterior, extending well past their normal entry point into the limb. By St 30 , neurites from segment 3 could be traced into the tail where they had branched within tail muscles or a small remnant of the caudilioflexorius muscle. Hamburger (1958) has also noted growth into the tail under these conditions.

Therefore, even though some mesenchyme usually remained distal to the plexus (see, for example, Fig. 2), in the complete absence of limb structures, neurites failed to leave the plexus to invade this mesenchyme and grew posteriorly instead. This bias for posterior growth appeared to be overcome in two situations. The first occurred when remnants of muscle remained in the limb. In such cases muscles were always innervated (see Figs. 3, 7, and 8), and in some of these cases neurons had to grow anteriorly to reach the muscle. For example, segment 3 neurites in the embryo shown in Figure $7 A$ traveled anteriorly to project to the dorsal crural nerve trunk, their normal projection pathway. This suggests that some cues originating from the target or target-associated mesenchyme are capable of overriding the bias for posterior growth. They might do this either by providing a specific attractant or at the minimum by altering the adjacent mesenchyme to provide an environment permissive for neurite elongation. The latter possibility is consistent with the observation that the organization of mesenchyme is dependent upon muscle (Kieny, 1983).

Although we do not have enough evidence to completely
A stage 25

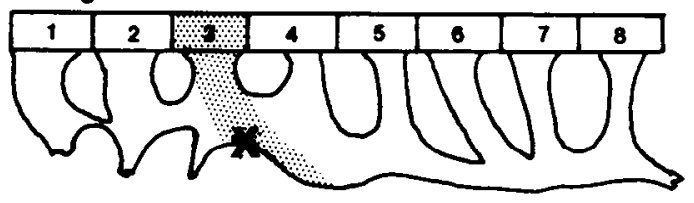

B stage 27

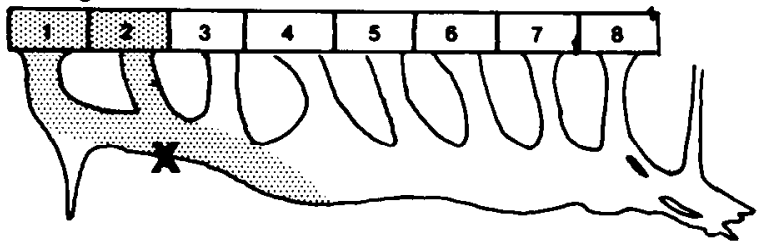

C stage 30

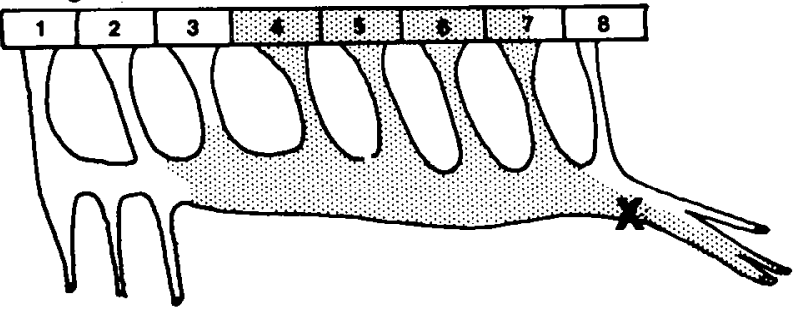

Figure 6. Neurites grow posteriorly in the absence of muscle remnants. In radical leg ablations, the spinal nerves met in the plexus area to form a "neuroma." The only peripheral nerves were small axial nerves that often formed opposite to segments 1 to 3 and entered the body wall, ventral to the leg rudiment. To determine whether there was any order in this simple pattern of nerve outgrowth, we injected HRP into the plexus region or spinal cord. We found that, at all stages, neurites grew toward the posterior within the plexus region. Leg muscles were always absent in these cases (see Fig. $3 A$ ) and in some cases, the pelvic girdle was also totally removed. Injection sites are indicated by $X$; labeled neurites are indicated by stippling. The A-P distribution of labeled somata in the cord is indicated by stippling above the ventral roots. $A$, In a St 25 embryo, injection of IIRP into the plexus regionjust posterior to LS3 labeled neurites from LS3. The labeled neurites extended approximately $100 \mu \mathrm{m}$ toward the posterior within the plexus region. Neurites from LS4 were not labeled: these have not traveled toward the anterior. $B$, In a St 27 embryo, neurites from $\mathrm{LS} 1$ and 2 have traveled up to $400 \mu \mathrm{m}$ toward the posterior. Neurites from levels posterior to the site of injection were not labeled. $C$, By St 30 , injection into the posterior portion of the plexus labeled neurites within the plexus up to level LS3 and motoneurons within the cord to level LS4. Neurites have traveled up to $900 \mu \mathrm{m}$ toward the posterior. This is a minimum estimate, since the HRP label will only travel a limited distance.

distinguish between these possibilities, our results favor some specific form of attraction. First, segmentally inappropriate spinal nerves were never observed to innervate muscle remnants $(N=14)$. For example, neurites from inappropriate segments 4 to 8 , shown in a second embryo with anterior muscle remnants (Fig. $7 B$ ), did not grow anteriorly to project to these muscle remnants, as did appropriate segment 3 neurites in theembryo shown in Figure $7 A$. Clearly, growth to muscle remnants was not a nonspecific response to the presence of noninnervated muscle. Furthermore, in the few cases in which we were able to retrogradely label the neurites projecting to a muscle remnant $(N=3)$, they arose from the correct motoneuron pool. In the example shown in Figure $8 A$, the sartorius remnant is innervated by motoneurons from the appropriate segments (LS 1 and 2 but not LS 3). Moreover, a reconstruction from sections of the cord in the transverse plane (Fig. 8B) shows that the neurons projecting to this remnant (right side) were located laterally in the normal sartorius pool location. 
More medial motoneurons that would normally project to ventral thigh muscles, and which were labeled by injecting the obturator nerve in the left (control) limb of the same embryo, did not project into this nerve trunk and presumably remained
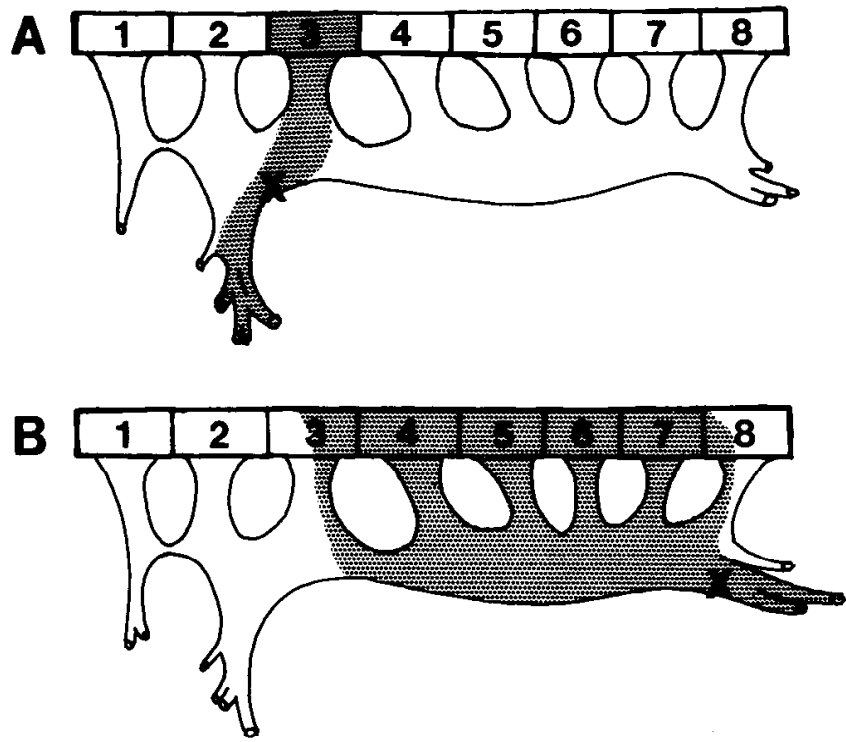

Figure 7. Direction of neurite growth in the plexus region when muscle remnants are present. $A$, Neurites turned toward the anterior in leg ablated embryos when portions of the target remained. In the embryo shown here, muscle remnants were present in the dorsal anterior limb, and a dorsal crural nerve trunk formed. HRP injected at level LS3 $(X)$ labeled neurites that traveled toward the anterior and entered the crural trunk as they normally would. Label is indicated by stippling, and segments are identified by numbers above the nerve pattern. $B$, Nonspecific chemotaxis toward remaining muscle tissue cannot explain the anomalous posterior growth of neurites in the plexus region. In this embryo, only dorsal anterior muscle remnants were present, and a dorsal crural nerve trunk formed, yet neurites from segments LS3 to 8 traveled within the plexus toward the posterior. HRP injected into the plexus region at the posterior, where neurites had entered the tail, labeled neurites from segments LS3 to 8 that would normally enter the sciatic nerve trunk. Similar results were obtained with cord injections. Label is indicated by stippling. within the plexus and grew posteriorly. We were able to make similar observations for two additional embryos. In summary, then, muscle remnants appear to provide a stimulus for neurite growth into the limb remnant, and this stimulus may be specific.

However, as noted earlier, muscle tissue was not always required for neurite growth into the limb remnant. In 11 cases an incipient obturator nerve formed even though no ventral thigh muscle, its normal target, was present. The obturator nerve shown in Figure 9 ended blindly in undifferentiated mesenchyme. Therefore, neurites can be caused to leave the plexus by the presence of permissive pathways (in this case presumably supplied by the ventral girdle) and/or by the presence of target or target-associated mesenchyme.

An important final point, illustrated by the embryo shown in Figure 9, is that although muscle targets appear to provide a specific stimulus to neurons, the normal sorting out of axons that takes place in the plexus region can occur in the complete absence of differentiated limb muscle. Normally, the intermingled axons of medial and lateral motoneurons sort out in the plexus in such a way that medial motoneurons take up a ventral position and lateral motoneurons take up a dorsal one (LanceJones and Landmesser, 1981a). If targets in the limb proper (either muscle or connective tissue components) provided cues necessary for sorting out at this point, we would expect that, in embryos with complete limb ablations, axons would fail to sort out and would enter any permissive pathways indiscriminately. This was not the case. Only axons from appropriate medial motoneurons entered the obturator nerve in these cases $(N=3$; see Fig. $9 B)$. Axons from these neurons (labeled by HRP injection into the incipient obturator nerve) were distributed throughout the D-V extent of the plexus proximally (Fig. $9 C$ ) but, within less than $200 \mu \mathrm{m}$, had come to occupy an extreme ventral position (Fig. $9 D$ ). They ended blindly in undifferentiated mesenchyme, which extended for some distance distally (Fig. 9, $E$ and $F$ ). This result shows that the cues necessary for growth cones to make this $\mathrm{D}-\mathrm{V}$ choice are contained locally in the mesenchyme at the limb base and need not arise from more distant structures including the target muscles. We obtained similar results in two additional embryos in which the obturator nerve was labeled. We cannot, of course, exclude the presence of a small number of undifferentiated

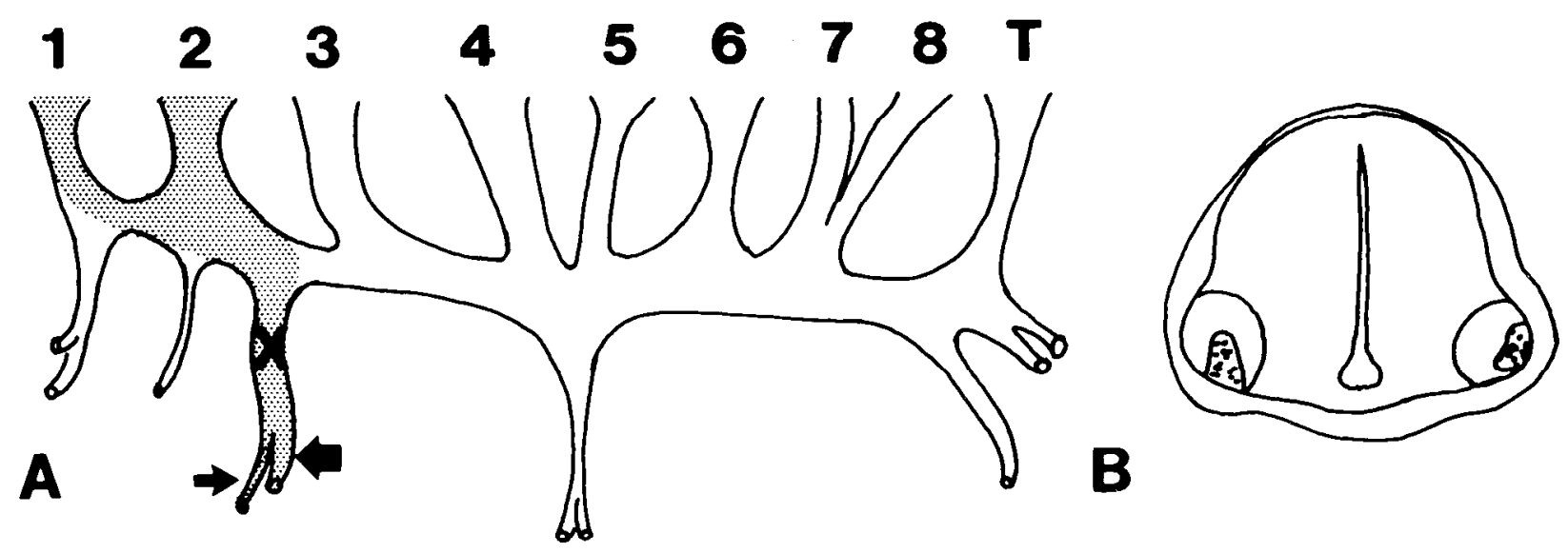

Figure 8. Specific innervation in partial leg ablations. A, Reconstruction of a St 29 embryo in which the obturator nerve is absent and the dorsal crural trunk is represented by a peripheral branch (large arrow) to a small remnant of the sartorius muscle. This nerve was injected with $\mathrm{HRP}$ at $X$. Stippling shows labeled neurites extending from the segments that normally innervate the sartorius muscle, LS1 and 2. The remaining features of this nerve pattern are axial nerves that leave the plexus area opposite LS1 and 2, a small cutaneous nerve that branches from the injected nerve (small arrow), a novel central nerve opposite LS4, and branches from the posterior plexus region into tail segments. $B$, A camera lucida reconstruction shows the distribution of motoneurons in the transverse plane of cord segments LS1 and 2 that have been labeled by the injection. On the experimental side (at right), labeled somata lie laterally, in the correct position for motoneurons innervating the sartorius muscle. On the left, motoneurons are labeled from an injection into the obturator nerve on the control side of the embryo and lie in a complementary medial position in the cord. 

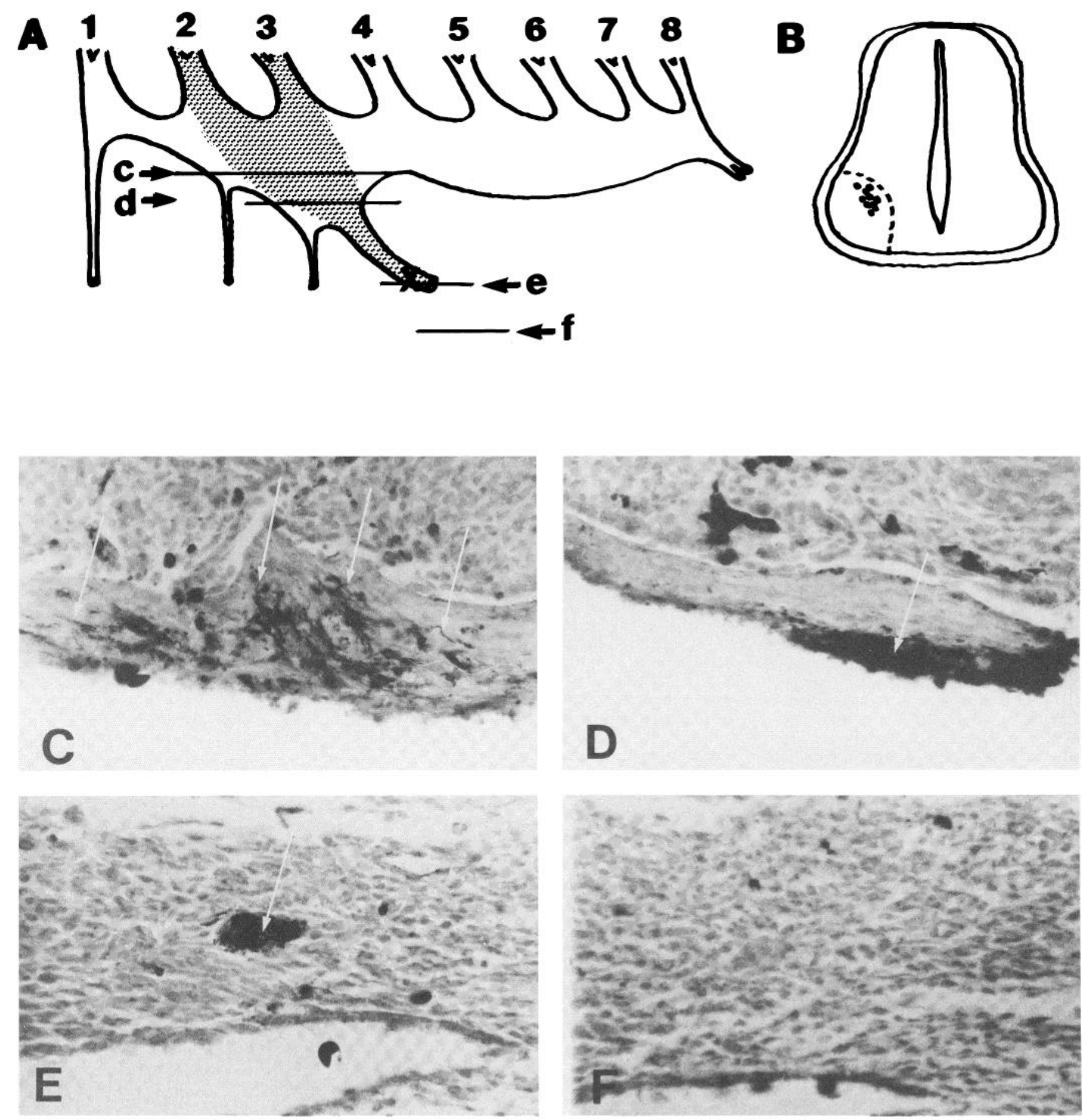

Figure 9. The sorting out of motoneurons in the absence of their target. In this St 29 embryo all leg muscles are absent. The nerve pattern $(A)$ is typical of a radical leg ablation, with the majority of the neurites remaining in the plexus area. However, an obturator nerve was sufficiently separated from the plexus to be injected with HRP $(X)$. Labeled neurites are indicated by stippling. All labeled somata lay in the correct pool position for obturator motoneurons, both in the A-P axis shown in $A$ and in the medial-lateral axis of the spinal cord shown in $B$, where the position of labeled somata was marked on camera lucida tracings through appropriate cord segments. Arrows $c$ to $f$ (in $A$ ) indicate the level of the sections shown in $C$ to $F$. Dark cells in the mesenchyme are red blood cells. Cross-sections through the plexus region at $c$ and $d$ show that the obturator neurites sorted normally within the plexus. They entered the plexus in a mixed state $(C$; arrows $)$ and, within $100 \mu \mathrm{m}$, gathered into the ventral portion of the plexus $(D$; arrow) before entering the obturator nerve. The labeled nerve is shown near its endpoint in $E$ (arrow); the mesenchyme $150 \mu \mathrm{m}$ distal to the nerve end, shown in $F$, contains no muscle remnants. This mesenchyme lies just distal to the pelvic girdle precursor. The more distal tissues, including the area where the target normally develops, have been removed.

premuscle cells. However, muscle does not normally occur in the position where these obturator nerves ended; the target is situated more distally. In addition, even small muscle remnants were detectable by the staining procedures we used at these stages. It was clear that the normal muscles for these nerves were absent.

It is possible that axons sorted out along the D-V axis in most or all of our experimental embryos. However, we could 
only assay this in those cases where nerves formed which were distinct enough to be selectively injected with HRP. Cues for A-P sorting may also be local and target independent, but for similar reasons we were unable to determine this directly.

Cell death in the absence of the periphery. Our results confirm the increased motoneuron cell death in embryos deprived of the periphery, as shown earlier by Hamburger (1975) and ChuWang and Oppenheim (1978). The lateral motor column on the operated side was noticeably smaller than the control side by St 30, and cell death was highest for motoneurons deprived of their target. For instance, in embryos without posterior muscle remnants, the reduction in cell number was most extensive in the posterior. Similar selective cell loss has been observed by others (Lance-Jones, 1982; Whitelaw and Hollyday, 1983a). The cross-sectional volume of the plexus declined concurrently. The specificity of innervation after cell death was no different from that assessed in early stages.

Although some cell death had taken place by St 28-29 on the experimental side (see Oppenheim et al., 1978), motoneurons deprived of their target were still numerous at that time. For instance, in Figure 9, somata of a medial motoneuron pool are labeled. A significant number of lateral motoneurons, which have been deprived of their target, have survived until St 29. If they had died, the lateral portion of the motor column would have been reduced in size, and the labeled somata would have come to lie closer to the white matter than normal.

\section{Discussion}

Neuronal pathfinding in the vertebrate limb has been postulated to involve several widely different mechanisms. Some have proposed that, for the most part, axons are passively deployed down limb-imposed pathways or channels (Summerbell and Stirling, 1981), the observed pattern being due primarily to the orderly outgrowth of axons in space and time. Others have favored a more active process in which growth cones respond to specific environmental cues (Lance-Jones and Landmesser, 1980b, 1981b; Lamb, 1981; Ferguson, 1983). Although it is clear that elements in the limb may constrain or restrict the response of axons (Summerbell and Stirling, 1981) and that there clearly are limb-defined pathways, all of the evidence is compatible with the existence of specific environmental cues (Lance-Jones and Landmesser, 1980b, 1981b; Hollyday, 1981; Stirling and Summerbell, 1983; Whitelaw and Hollyday, 1983a, b).

The present paper addresses these two aspects of neuronal pathfinding. The first aspect is directed toward the nonspecific, limb-imposed pathways and asks, what elements within the limb lead to the development of a constant gross anatomical nerve pattern, regardless of the specificity of innervation? Second, are the cues that growth cones use to make specific decisions in the plexus region provided by local or distant limb tissues? Our results suggest that the local environment of growth cones is critical, both in providing permissive pathways and in determining that specific populations of growth cones take up characteristic positions within the pathways.

Permissive pathways. The gross anatomical nerve pattern in the chick hindlimb retains its general features even when incorrect neurites have been channeled into the limb by experimentally reversing the cord (Lance-Jones and Landmesser, 1980b, 1981b), by shifting or reversing the limb bud (LanceJones and Landmesser, 1981b; Summerbell and Stirling, 1981; Ferguson, 1983), or by adding a supernumerary limb bud (Morris, 1978; Hollyday, 1981). These results suggest that there are permissive and probably preferred pathways in the embryonic environment that motoneuron growth cones will follow, regardless of their identity.

We have shown that the major pathways in the limb develop independently of one another and that, when one pathway is removed in partial leg ablations, remaining pathways are still formed. These results suggest that the elements determining the gross anatomical pattern are local and that one determinant can be removed without greatly altering the pattern at other positions.

One local element, the central portion of the pelvic girdle, is important in the allocation of neurites into anterior and posterior nerve trunks. When it is absent, neurites move directly into adjacent leg muscles in the region between the normal nerve trunks. This pattern is also seen when a gap is made in the central girdle region during preparation of double dorsal limbs (C. Lane-Jones, personal communication). During normal development, the precursor of the pelvic girdle is present as a precartilage condensation at the time of nerve trunk formation (K. W. Tosney and L. T. Landmesser, unpublished observations) and probably provides a nonpermissive environment for the advancement of growth cones. Neurite outgrowth is then restricted to the plexus region and the anterior and posterior gaps within the girdle, through which the major nerve trunks enter the leg.

The obturator nerve also avoids traversing pregirdle tissue, traveling adjacent to the girdle and entering the middle of the leg from the girdle's ventral margin. Mesenchyme lying close to the ventral girdle may, in part, provide the pathway used by these neurites.

Other precartilage tissues, such as the femur, may normally provide a nonpermissive environment as well. However, the femur is too distant from the plexus to affect the routes that growth cones take within the plexus region, and it plays no significant role in the separation of anterior and posterior nerve trunks.

Posterior growth in the plexus region. When the limb was either partly or totally ablated, we found that most axons grew toward the posterior within the plexus region. Similar growth toward the posterior was observed when the limb bud was shifted short distances anteriorly (Lance-Jones and Landmesser, 1981b); anterior spinal nerves, that would have entered the crural trunk, now grew posteriorly (sometimes for up to three segments) and only emerged from the plexus region when they encountered the region of the sciatic trunk. More posterior sciatic segments (LS5 to 8) failed to project to the limb and instead grew posteriorly toward the tail, as in the present experiments. Such posterior growth appears to be correlated with the absence of a suitable distal pathway for axon elongation into the limb. In leg ablations such pathways have been physically removed; in limb shifts, the position of the permissive distal pathways is altered and axons encounter a nonpermissive environment that does not allow axon elongation, the central pelvic girdle region. If the only areas permissive for axon elongation into the limb were the small gaps in the girdle through which the crural and sciatic trunks normally pass, the nerve pattern observed with limb shifts would be expected.

We do not understand why axons should grow to the posterior, as opposed to the anterior, in these cases. The limb girdle per se cannot be the cause, because posterior growth occurs even when the limb girdle is totally lacking. It is possible that slight differences in the timing of axon ingrowth along the A$P$ axis (see, for example, Lance-Jones and Landmesser, 1981a) may contribute to this posterior bias. Since anterior spinal nerves slightly precede more posterior ones into the plexus region, when deprived of any chance of distal growth, axons may turn posteriorly because this region contains fewer axons to constrain or interfere with their elongation.

Of more interest is a consideration of the situations in which this posterior bias is overcome, since this should provide us with information about the axonal guidance features which operate during normal development. In some cases a nonspecific permissive pathway may suffice. For example, in the limb 
shift experiments, posteriorly growing axons from LS 1 and 2 are able to leave the plexus when they encounter the region of the sciatic trunk, even though this is a totally inappropriate pathway and does not contain the specific cues these axons normally use to achieve their specific projections (Lance-Jones and Landmesser, 1981b). However, the present experiments suggest that specific cues present in the target or target-associated mesenchyme may also encourage axons to leave the plexus region.

Specificity. A number of previous studies on vertebrate limbs strongly suggest that specific chemical cues of some sort are required to explain axon behavior both during normal development (Landmesser, 1978b; Lance-Jones and Landmesser, 1981a) and following experimental manipulations (LanceJones and Landmesser, 1980a, b, 1981b; Lamb, 1981; Ferguson, 1983; Whitelaw and Hollyday, 1983a, b).

The present study, designed to obtain additional information on the nature of such cues, has shown that in all cases they appear to be quite local. This is consistent with several previous studies on the chick limb system (Lance-Jones and Landmesser, 1981b; Whitelaw and Hollyday 1983b). Our observations that axons sort out on the $\mathrm{D}-\mathrm{V}$ axis in the complete absence of limb muscle and other structures shows that these cues must be contained locally within the mesenchyme at the base of the limb at the time of plexus formation (Sts 22 to 24). Whether these cues arise locally as this tissue differentiates or are imparted by premuscle cells of somitic origin that migrate through the plexus region into the limb (Chevallier et al., 1978) remains to be determined. When such premuscle cells were killed by somite irradiation, Lewis et al. (1981) observed gross anatomical disruption of the plexus region (i.e., lack of segmentation). However, they did not determine whether normal D-V sorting takes place in such muscleless limbs, a distinct possibility, even though muscle nerves did not form.

Once having sorted out in the plexus region, axons might elongate nonselectively down permissive pathways, their subsequent deployment to specific muscles being determined solely by the spatial position they occupy. For example, lateral motoneurons normally may never project out of the obturator nerve, because this nerve emerges from the ventral plexus, opposite to the dorsal position they have come to occupy. However, it is probable that additional specific cues exist in the limb proper and may be used as axons grow down major nerve trunks and form muscle nerves.

The existence of such cues is suggested by the results of experiments using double dorsal limbs (Lance-Jones, 1982; C. Lance-Jones, personal communications) and limb shifts (Lance-Jones and Landmesser, 1981b). In both cases, axons growing down foreign nerve trunks are apparently enticed to leave these permissive pathways by cues from their normal targets (see also Summerbell and Stirling, 1981). They project to such targets by taking routes through normally less permissive mesenchyme. The specificity of innervation observed in serially duplicated chick limbs (Whitelaw and Hollyday, 1983b) would also seem to require specific cues within the limb.

In our experiments muscle remnants were innervated appropriately, suggesting that such cues are quite specific, possibly to the level of the individual muscle. Clearly, innervation of such remnants was not a nonspecific response of axons to denervated muscle, mediated by some chemical factor or sprouting agent. However, at first it seems difficult to reconcile the fact that foreign axons were never found to project to such muscle remnants, with the fact that axons have been shown to effectively innervate foreign muscles in a variety of other circumstances (Morris, 1978; Hollyday, 1981; Lance-Jones and Landmesser, 1981b; Summerbell and Stirling, 1981; Landmesser and O'Donovan, 1984).

One explanation is that, even though foreign axons will readily elongate down permissive pathways, additional spēcific cues may enhance the growth of correct axons and would be required for axons to deviate from these paths into less permissive mesenchyme. In our experiments, we may have removed all of the permissive pathways in the limb proper so that axons would have to traverse less preferred mesenchyme to reach their targets. For instance, when only a sartorius muscle remnant is left in the anterior leg, axons from LS3 and other pools in LS1 and 2 do not form obturator or crural nerves: only sartorius axons traverse the gap in the central pelvic girdle and enter the leg (see Fig. 8). Since normal obturator and crural nerve trunks can be formed even by foreign axons channeled into the crural plexus, it is reasonable to assume that the normal crural trunk pathway within this leg has been removed. Sartorius axons, then, would innervate the leg in response to specific cues that may emanate from their target. Since target remnants were all from thigh muscle and relatively close to the plexus region, we estimate that they should be within filopodial grasp of individual growth cones (Tosney and Landmesser, 1983). The local nature of specific cues, whether in the plexus or the limb per se, has been suggested by several other studies in this system (Lance-Jones and Landmesser, 1981b; Summerbell and Stirling, 1981; Lance-Jones, 1982) and is consistent with the emerging view of how developing arthropod neurons establish both central (Raper et al., 1983) and peripheral (Bentley and Keshishian, 1982; Ho and Goodman, 1982) projections.

Finally, it is possible that muscle nerves fail to form in muscleless limbs (Lewis et al., 1981) because permissive pathways from the major nerve trunks to muscles fail to form. Such permissive pathways might require factors from muscle and/or blood vessels and organized connective tissue normally associated with muscle.

Conclusions. We conclude that two types of interactions between the growth cone and its environment govern pathfinding in the chick hindlimb. One class of interaction is a nonspecific one, in that the identity of the growth cone is not called into play. It can be thought of as the extension of growth cones along permissive pathways in the embryonic environment and a failure to extend into nonpermissive regions such as the central girdle area. Although many other regions of the limb may be permissive, in the absolute sense that axons can grow through them, axons are normally restricted to certain more permissive areas that define the major nerve trunks and muscle nerves. These more permissive or preferred pathways are traversed equally well by all species of lumbosacral motoneurons (Lance-Jones and Landmesser, 1981b; Landmesser and O'Donovan, 1984) and are thus, by definition, nonspecific.

The other class of interactions involves specific and local cues, which are required to explain the highly specific projection patterns normally observed. We have shown that such cues used by axons to sort out in the plexus region need not arise from limb tissue. However, it is probable that limb tissue, including muscle, also contains specific cues. These may be distributed along the permissive pathways normally used by a subset of axons, encouraging continual adjustments in position. Alternatively, having sorted out in the plexus area, axons may be passively guided until they form muscle nerves, when specific cues would again come into play. The existence of specific chemical cues for both axonal guidance and target selection has been supported by studies in other systems as well (Purves et al., 1981; Wigston and Sanes, 1982; Raper et al., 1983). However, the further elucidation of such cues will probably require a more simplified in vitro model similar to that recently developed for the retinotectal system (Bonhoeffer and Huf, 1982).

\section{References}

Bennett, M. D., D. Davey, and K. Uebel (1980) The growth of segmental nerves from the brachial myotomes into the proximal muscles of the chick forelimb during development. J. Comp. Neurol. 189: 335-357. Bentley, D., and H. Keshishian (1982) Pathfinding by peripheral pi- 
oneer neurons in grasshoppers. Science 218: 1082-1088.

Bonhoeffer, F., and J. Huf (1982) In vitro experiments on axon guidance demonstrating an anterior-posterior gradient on the tectum. EMBO J. 4: 427-431.

Chevallier, A., M. Kieny, and A. Maugen (1978) Limb-somite relationship: Effect of removal of somitic mesenchyme on the wing musculature. J. Embryol. Exp. Morphol. 43: 263-278.

Chu-Wang, I. G., and R. W. Oppenhiem (1978) Cell death of motoneurons in the chick embryo spinal cord. II. A qualitative and quantitative analysis of degeneration in the ventral root including evidence for axon outgrowth and limb innervation prior to cell death. J. Comp. Neurol. 177: 59-86.

Derby, M. A., and J. E. Pintar (1978) Histochemical specificity of Streptomyces hyaluronidase and chondroitinase ABC. Histochem. J. 10: $529-547$.

Ferguson, B. A. (1983) Development of motor innervation of the chick following dorsal-ventral limb bud rotations. J. Neurosci. 3: 17601772.

Hamburger, V. (1958) Regression versus peripheral control of differentiation in motor hypoplasia. Am. J. Anat. 102: 365-410.

Hamburger, V. (1975) Cell death in the development of the lateral motor column of the chick embryo. J. Comp. Neurol. 160: 535-546.

Hamburger, V., and H. L. Hamilton (1951) A series of normal stages in the development of the chick embryo. J. Morphol. 88: 49-82.

Ho, R. K, and C. S. Goodman (1982) Peripheral pathways are pioneered by an array of central and peripheral neurons in grasshopper embryos. Nature 297: 404-406.

Hollyday, M. (1981) Rules of motor innervation in chick embryos with supernumerary limbs. J. Comp. Neurol. 202: 439-465.

Kieny, M. A. (1983) Cell and tissue interactions in the organogenesis of the avian limb musculature. In Limb Development and Regeneration, Part B, J. F. Fallon and A. I. Caplan, eds., pp. 293-302, Alan R. Liss, Inc., New York.

Lamb, A. H. (1981) Selective bilateral motor innervation in Xenopus tadpoles with one hindlimb. J. Embryol. Exp. Morphol. 65: 149-163.

Lance-Jones, C. (1982) Motoneuron connectivity in embryonic chick limbs with an altered complement of muscle. Soc. Neurosci. Abstr. $8: 435$.

Lance-Jones, C., and L. Landmesser (1980a) Motoneurone projection patterns in embryonic chick limb following partial deletions of the spinal cord. J. Physiol. (Lond.) 302: 559-580.

Lance-Jones, C., and L. Landmesser (1980b) Motoneurone projection patterns in the chick hindlimb following early partial reversals of the spinal cord. J. Physiol. (Lond.) 302: 581-602.

Lance-Jones, C., and L. Landmesser (1981a) Pathway selection by chick lumbosacral motoneurones during normal development. Proc. R. Soc. Lond. (Biol.) 214: 1-18.

Lance-Jones, C., and L. Landmesser (1981b) Pathway selection by embryonic chick motoneurones in an experimentally altered environment. Proc. R. Soc. Lond. (Biol.) 214: 19-52.
Landmesser, L. (1978a) The distribution of motoneurons supplying chick hind limb muscles. J. Physiol. (Lond.) 284: 371-389.

Landmesser, L. (1978b) The development of motor projection patterns in the chick hindlimb. J. Physiol. (Lond.) 284: 391-414.

Landmesser, L. (1980) The generation of neuromuscular specificity. Annu. Rev. Neurosci. 3: 279-302.

Landmesser, L., and D. G. Morris (1975) The development of functional innervation in the hind limb of the chick embryo. J. Physiol. (Lond.) 249: $301-326$.

Landmesser, L., and M. J. O'Donovan (1984) The activation patterns of embryonic chick motoneurons projecting to inappropriate muscles. J. Physiol. (Lond.) 347: 205-224.

Lewis, J., A. Chevallier, M. Kieny, and L. Wolpert (1981) Muscle nerves do not develop in chick wing devoid of muscle. J. Embryol Exp. Morphol. 64: 211-232.

Morris, D. G. (1978) Development of functional motor innervation in supernumerary hindlimbs of the chick embryo. J. Neurophysiol. 41 : 1450-1465.

Oppenheim, R. W., I. -W. Chu-Wang, and J. L. Maderdrut (1978) Cell death of motoneurons in the chick embryo spinal cord. III. The differentiation of motoneurons prior to their induced degeneration following limb bud removal. J. Comp. Neurol. 177: 87-112.

Purves, D., W. Thompson, and J. Yip (1981) Re-innervation of ganglia transplanted to the neck from different levels of the guinea-pig sympathetic chain. J. Physiol. (Lond.) 313: 49-63.

Raper, J. A., M. Bastiani, and C. S. Goodman (1983) Pathfinding by neuronal growth cones in grasshopper embryos. I. Divergent choices made by the growth cones of sibling neurons. J. Neurosci. 3: 20-30.

Romer, A. S. (1927) The development of the thigh musculature of the chick. J. Morphol. 43: 347-385.

Stirling, R. V., and D. Summerbell (1983) Familiarity breeds contempt: the behavior of axons in foreign and familiar environments. In Limb Development and Regeneration, Part A, J. F. Fallon and A. I. Caplan, eds., pp. 217-229, Alan R. Liss, Inc., New York.

Summerbell, D., and R. V. Stirling (1981) The innervation of dorsoventrally reversed chick wings: Evidence that motor axons do not actively seek out their appropriate targets. J. Embryol. Exp. Morphol. 61: 233-247.

Tosney, K. W., and L. T. Landmesser (1983) Growth cone guidance in the chick hindlimb. Soc. Neurosci. Abstr. 9: 210.

Whitelaw, V., and M. Hollyday (1983a) Thigh and calf discrimination in the motor innervation of the chick hindlimb following deletions of limb segments. J. Neurosci. 3: 1199-1215.

Whitelaw, V., and M. Hollyday (1983b) Position-dependent motor innervation of the chick hindlimb: Serial and parallel duplications of limb segments. J. Neurosci. 3: 1216-1225.

Wigston, D. J., and J. R. Sanes (1982) Selective reinnervation of adult mammalian muscles by axons from different segmental levels. $\mathrm{Na}$ ture 299: 464-467. 